\title{
Percepción del estado de salud bucal de adolescentes y adultos jóvenes en tratamiento por drogodependencia
}

\author{
Enrique Rotemberg ${ }^{1}$ \\ Inés Salveraglio ${ }^{1}$ \\ Sylvia Piovesán ${ }^{1}$ \\ María Teresa Almaráz ${ }^{2}$ \\ Beatriz Ferreira ${ }^{2}$ \\ Karinna Smaisik' \\ María del Cármen Mazzuco ${ }^{3}$
}

Oral status perception by individuals in drug dependence

\section{Resumen}

Objetivo general: conocer la percepción de adolescentes y adultos jóvenes vinculados al consumo abusivo de drogas a partir de la situación de dependencia. Materiales y métodos: se planteó un modelo de investigación cualitativo. La muestra teórica por saturación fue población adolescente y adulta joven que se asistió en régimen de internación en el Portal Amarillo. Criterios de inclusión: a) tener entre 15 y 24 años, b) estar en tratamiento por consumo de drogas. Criterios de exclusión: a) pacientes no lúcidos, b) pacientes no colaboradores. Resultados: los participantes refieren como salud bucal tener los dientes limpios, blancos y alineados; como enfermedad caries, sangrado de encías y enfermedades transmisibles; y como funciones alteradas la masticación, la estética y el gusto. Antes de la situación de dependencia la higiene bucal era regular, después de las comidas y con instrucción recibida, a partir de la dependencia la higiene fue muy irregular, si se acordaban y sin refuerzo de la instrucción. Previo al consumo eran individuos integrados al estudio o al trabajo, con vida familiar ordenada, con pareja o en procura de tenerla; posteriormente al consumo relatan automarginación con pérdida de contacto con la pareja e hijos, aislamiento de la familia y discriminación social. El consumo dificulta el acceso a la atención odontológica, excepto en situaciones de urgencia. Conclusiones: Las personas jóvenes en tratamiento por dependencia a las drogas perciben el deterioro de su salud bucal a partir de la situación de consumo, alterando la estética, las funciones orales, la autoestima y la calidad de vida.

Palabras clave: salud bucal, drogodependencia, adolescente, adulto joven.

Facultad de Odontología FOUdelar, Montevideo, Uruguay.

Programa APEX- Cerro - Universidad de la República.

Programa Minessota (Montevideo) y Junta Nacional de Drogas (Presidencia de la República), Uruguay. 


\section{Abstract}

General objective: to know the perception of adolescents and young adults linked to the abusive consumption of drugs as a result of a dependency situation.

Material and method: a qualitative research model was proposed. The theoretical sample by saturation was of adolescent and young adult population that was attended in Portal Amarillo. Inclusion criteria: a) to be between 15 and 24 years old, b) to be in treatment for drug use. Exclusion criteria: a) non-lucid patients, b) non-collaborating patients.

Results: Participants refer to oral health as clean, white and aligned teeth; as cavities disease, gum bleeding and communicable diseases; and as altered functions chewing, aesthetics and taste. Prior to the dependency situation oral hygiene was regular, after meals and with received instructions. After the dependence the hygiene was very irregular, only if they remembered to do it and without instruction reinforcement. Before consumptions they were individuales integrated into the study or work, with an ordered family life, with a partner or searching for one. After consumption, they reported self-marginalization, loss of the partner and contact with the children, isolation of the family and social discrimination. Consumption makes it difficult to access dental care, except in emergency situations.

Conclusions: Young people in treatment for drug dependency perceive the deterioration of their oral health from the situations of consumption, altering the esthetic, oral functions, selfesteem and quality of life.

Keywords: oral health, drug dependence, adolescent, young adult.

Este artículo es una republicación del artículo:

Rotemberg E, Salveraglio I, Piovesán S, Almaráz M, Ferreira B, Smaisik K, Mazzuco M. Percepción del estado de salud bucal de adolescentes y adultos jóvenes en tratamiento por drogodependencia. JOralRes [Internet]. 31 de octubre de 2019 [citado 20 de noviembre de 2020];8(5):378-85. Disponible en: https://revistas.udec.cl/index.php/journal_of_oral_research/article/view/1877

\section{Introducción}

Según la Organización Mundial de la Salud (OMS): "Droga es toda sustancia terapéutica o no que, introducida en el organismo por cualquier vía de administración (inhalación, ingestión, fricción, administración parenteral, endovenosa), produce una alteración, de algún modo, del natural funcionamiento del sistema nervioso central del individuo y es, además, susceptible de crear dependencia psicológica, física o ambas" ${ }^{(1)}$.

El consumo de sustancias psicoactivas (SPA), conocidas comúnmente como drogas psicoactivas, puede modificar la conciencia, el estado de ánimo o los procesos de pensamiento de un individuo ${ }^{(2)}$. Muchas de estas sustancias han sido utilizadas en la historia de la humanidad con fines religiosos, culturales, recreativos $y$ medicinales. Todo consumo de sustancias, sean legales o ilegales, naturales o sintéticas, conlleva riesgos diferenciados según edad, sexo, formas y frecuencia de uso, contextos y circunstancias personales ${ }^{(3)}$. El abuso de sustancias se refiere en general a los problemas relacionados con el consumo de SPA ${ }^{(4)}$.

Existen diferentes pautas de consumo, incluyendo el consumo experimental, ocasional, habitual, hasta situaciones de abuso y dependencia. Cualquier tipo de consumo puede ser problemático (3). En un inicio prevalece el efecto positivo y placentero donde el consumo está bajo control del usuario ${ }^{(5,6)}$. Gradualmente en algunas personas 
la motivación del consumo va cambiando y para experimentar placer o evitar malestar aumenta la cantidad de sustancia y frecuencia de la dosis. Finalmente un porcentaje de la población pierde el control en el uso, se torna compulsivo e incapaz de abandonar las drogas pese a conocer las consecuencias ${ }^{(6)}$. El policonsumo de drogas es una modalidad de consumo que plantea mayores riesgos debido a la combinación de diversas SPA (7). Las pautas de consumo varían según la oferta del mercado de drogas, la búsqueda de cambio y el contexto relacional ${ }^{(8)}$.

El abuso de drogas ha sido definido como "la utilización excesiva, persistente o esporádica de un fármaco de forma incongruente o desvinculada con la práctica médica admisible" (4). El término farmacodependencia se amplió al concepto de drogodependencia ${ }^{(9)}$. La OMS recomendó sustituir la expresión drogadicción o habituación a drogas por dependencia a las drogas, dada la connotación despectiva del término drogadicto ${ }^{(4,9)}$.

El panorama mundial del consumo de drogas resulta difuso porque muchas personas que consumen, sea de manera ocasional o habitual, tienden a ser policonsumidoras utilizando más de una sustancia simultánea o alternadamente ${ }^{(10)}$. La cadena de consumo en adolescentes frecuentemente se inicia con el alcohol y el tabaco, seguida por las drogas ilícitas ${ }^{(11,12)}$. Las drogas ilegales más consumidas son el cannabis, los estimulantes tipo anfetamínicos, los opioides y las sustancias relacionadas con la cocaína (10). Los patrones de drogas usadas incluyen inyección de opioides, anfetaminas y derivados de cocaína, con riesgo de contraer y contagiar enfermedades transmisibles ${ }^{(13)}$.

Situación epidemiológica actual del Uruguay:

La Séptima Encuesta Nacional sobre Consumo de Drogas en Estudiantes de Enseñanza Media en Uruguay del año 2016 destaca: a) el alto consumo de alcohol y bebidas energizantes con elevado contenido de cafeína, seguido por tabaco y marihuana, y en menor porcentaje tranquilizantes sin prescripción médica, cocaína, alucinógenos y estimulantes tipo anfetamínico ${ }^{(14)}$.

Los indicadores de percepción de riesgo incorporados en la presente encuesta, según las declaraciones de los estudiantes, identifican la percepción de riesgo alta como factor de protección, mientras que una baja percepción de riesgo incrementa la probabilidad de uso/abuso de alguna sustancia. En general se constató que las sustancias asociadas a menor riesgo fueron el alcohol, el tabaco y la marihuana, tanto en consumos ocasionales como frecuentes ${ }^{(14)}$.

La edad promedio de inicio de consumo varía con el sexo, la sustancia y el ámbito de residencia (Montevideo e interior) (Tabla 1) ${ }^{(14)}$.

\begin{tabular}{|l|c|c|c|c|c|c|}
\hline Sustancia & General & Desvío típico & Hombre & Mujer & Montevideo & Interior \\
\hline Alcohol & 12,75 & 2,3 & 12,45 & 13,03 & 12,66 & 12,82 \\
\hline Tranquilizantes sin prescripción médica & 13,40 & 2,79 & 12,77 & 13,75 & 13,41 & 13,40 \\
\hline Tabaco & 14,14 & 1,91 & 13,91 & 14,30 & 14,03 & 14,23 \\
\hline Marihuana & 14,84 & 1,56 & 14,72 & 14,95 & 14,71 & 14,98 \\
\hline Cocaína & 15,50 & 1,69 & 15,57 & 15,39 & 15,68 & 15,34 \\
\hline Alucinógenos & 15,95 & 1,9 & 15,92 & 15,97 & 15,91 & 15,98 \\
\hline Éxtasis & 16,18 & 1,42 & 16,32 & 16,01 & 16,05 & 16,30 \\
\hline
\end{tabular}

Base: Consumidores alguna vez en la vida de cada sustancia

Tabla 1. Edad inicio promedio por sustancia según sexo y ámbito de residencia. Estudiantes de Enseñanza Media. Encuesta Uruguay 2016 (\%) ${ }^{(14)}$ 
La Sexta Encuesta Nacional en Hogares sobre Consumo de Drogas mostró que los varones presentaron prevalencia de consumo superior a las mujeres para todos los rangos etarios, aunque la brecha es menor en la población más joven, excepto para el consumo de tranquilizantes, hipnóticos y antidepresivos con o sin indicación médica ${ }^{(15)}$.

El Gobierno de Uruguay en el año 2013 promulgó una ley para reglamentar el cultivo de la planta de cannabis y la producción, venta y consumo en forma recreativa. Desde entonces, el Gobierno de nuestro país ha promulgado otros decretos y ordenanzas concernientes a la aplicación de reglamentaciones sobre elementos específicos como el uso medicinal de cannabis, la comercialización y venta para uso recreativo en farmacias y la inscripción de los consumidores con fines recreativos en un registro ${ }^{(7)}$.

Las personas que consumen sustancias son en su mayoría jóvenes, entre los 16 y 25 años, siendo la iniciación y la expresión pública de esa conducta cada vez más temprana, incluso antes de los 14 años. El consumo problemático presenta factores de riesgo y factores de protección. Entre los factores de riesgo se destacan la naturalización del consumo en la cultura juvenil, la poca contención del sistema educativo que conduce al abandono de la educación formal y la ausencia de proyectos individuales sumado a la marginación social ${ }^{(16)}$.

El Informe Mundial sobre la Drogas de la UNODC del año 2009 indicó que la mayoría de las personas comenzaron a consumir drogas durante su juventud. Los jóvenes podrían responder a los cambios en la disponibilidad de drogas o a las percepciones sociales del consumo en mayor grado que las personas adultas. Además, el inicio precoz del consumo aumenta el riesgo de consecuencias sociales y sanitarias negativas sumado al vínculo con el delito bajo los efectos de las drogas o para financiar su obtención ${ }^{(17)}$.

El consumo de SPA afecta a todas las áreas de la salud: social, biológica, psicológica, cogniti- va, emocional y cultural ${ }^{(18)}$. Según la American Academy of Pediatric Dentistry (AAPD) la situación de abuso con frecuencia coexiste con condiciones psiquiátricas como depresión, ansiedad, déficit atencional, conducta desafiante, trastorno bipolar, estrés postraumático, bulimia nerviosa, fobia social y esquizofrenia ${ }^{(19)}$.

El empleo de drogas tiene un efecto negativo tanto en los hábitos de higiene como en la prevalencia de caries, gingivitis y periodontitis en relación a la población general ${ }^{(20-25)}$.

Entre los factores de protección para evitar el uso habitual y dependencia a las drogas se destacan los atributos propios del sujeto (voluntad, autoestima, proyecto de vida); la figura de la familia en su constitución y rol de sus integrantes; y la estabilidad de los vínculos sociales (educativo-laborales, culturales, familiares, de pareja) ${ }^{(16)}$.

La salud es considerada un evento dinámico denominado proceso-salud-enfermedad-atención (PSEA). Las representaciones del PSEA en los jóvenes incluye el componente bucal como parte de la salud general, no sólo por el dolor físico sino también por la importancia estética ${ }^{(26)}$. La salud bucodental está estrechamente relacionada con la salud general y la calidad de vida de las personas, al afectar las funciones orales y las interacciones sociales. Por ejemplo, la caries dental puede ser la causa de una masticación deficiente, disminución del apetito, problemas de sueńo y un menor rendimiento escolar y laboral ${ }^{(27)}$.

La salud bucodental es polifacética e incluye, entre otros aspectos, la capacidad de hablar, sonreír, oler, saborear, tocar, masticar, tragar y transmitir una serie de emociones a través de las expresiones faciales ${ }^{(28)}$.

Un estudio realizado en Cartagena de Indias, Colombia, demostró un impacto negativo en la calidad de vida relacionada con la salud oral en adultos jóvenes por: pérdidas dentarias al contar con menos de 19 piezas, presencia de restos radiculares, deposición de sarro y ausencia de servicios de salud $^{(29)}$. 
Objetivo general: Conocer la percepción de adolescentes y adultos jóvenes en tratamiento por consumo abusivo de drogas sobre su salud bucal a partir de la situación de dependencia a las drogas.

Objetivos específicos:

a) Estimar los conocimientos del proceso saludenfermedad-atención y expectativas sobre la situación bucal.

b) Identificar problemas de acceso a los servicios de atención odontológica en la población objeto.

\section{Materiales y métodos}

Se planteó un modelo de investigación cualitativa. Las técnicas utilizadas fueron entrevistas personales y grupos focales en jóvenes que se encontraban en régimen residencial en el Portal Amarillo en los tres meses del trabajo de campo. Dicha Institución es el Centro de Referencia Nacional de la Red Drogas desde el año 2006, combinando el régimen ambulatorio, centro diurno y residencial para la atención de pacientes usuarios de la Administración de los Servicios de Salud del Estado (ASSE) ${ }^{(30)}$. Se tuvo en cuenta la guía COREQ (criterios consolidados para informar y publicar sobre investigaciones cualitativas) y la lista de comprobación de acuerdo a los tres dominios, 1) investigadores, 2) diseño del estudio y 3) análisis y hallazgos.

Criterios de inclusión: a) estar comprendido en la franja etaria de 15 a 24 años, b) estar en tratamiento por consumo de drogas en régimen residencial.

Criterios de exclusión: a) pacientes no lúcidos, b) pacientes no colaboradores.

Resultaron en total 18 entrevistas y 3 grupos de discusión, interviniendo en total " 32 " participantes.

La modalidad de entrevista fue guionada en base a una experiencia piloto realizada en etapa previa con personas atendidas en el Portal Amarillo. En dicha experiencia también se ca- libraron los integrantes del equipo investigador en su roles de entrevistador u observador.

Toda instancia fue registrada en grabaciones sin identificar nombres de los participantes y además se tomó nota sobre su actitud e interés en la entrevista.

De acuerdo a ese interés que muchas veces se trasformó en aburrimiento, cansancio o silencio se debió recurrir a un complemento, con otra herramienta ya que las entrevistas duraban sólo entre 15 y 20 minutos. En este sentido se les solicitó dibujar la boca en estado de salud y enfermedad, bajo la supervisión exclusiva de educadores. La descripción y categorización de los dibujos estuvo a cargo de nuestro equipo.

Para el análisis de la información registrada se transcribieron, organizaron y ordenaron los datos obtenidos. Se construyeron categorías y subcategorías a partir de los textos desgrabados para analizar los testimonios orales.

Se estudió la percepción individual del estado de salud oral en el período anterior y posterior a la situación de dependencia a las drogas de acuerdo a la memoria y representaciones de cada sujeto.

Las categorías que surgieron luego de examinar el texto de las desgrabaciones fueron: conocimiento, higiene y relación con el entorno. De ellas se derivan subcategorías (Cuadro 1).

\begin{tabular}{|c|c|c|}
\hline Conocimiento & Higiene & $\begin{array}{c}\text { Relación con el } \\
\text { entorno }\end{array}$ \\
\hline Salud bucal & Realización & Trabaja y/o estudia \\
\hline $\begin{array}{c}\text { Enfermedades } \\
\text { bucales }\end{array}$ & Frecuencia & Relación de pareja \\
\hline Funciones orales & $\begin{array}{c}\text { Educación en } \\
\text { salud bucal }\end{array}$ & $\begin{array}{c}\text { Relación con la familia } \\
\text { y con la sociedad }\end{array}$ \\
\hline
\end{tabular}

Cuadro 1. Categorías y subcategorías

Consideraciones éticas: El protocolo de investigación fue aprobado por el Comité de Ética en Investigación de la Facultad de Odontología con el número de expediente 315/16. Los participantes fueron informados y se les solicitó la 
firma del consentimiento asegurándoles la absoluta privacidad de los datos y el anonimato.

\section{Resultados}

A partir de los datos cualitativos recogidos y analizados así como la información recabada en las historias clínicas de los 32 pacientes surgen los siguientes hallazgos.

El promedio de edad de los jóvenes fue de 20 años con predominio de jóvenes del género masculino en terapia en el Portal Amarillo, en coincidencia con datos epidemiológicos hallados en el mismo centro asistencial que probablemente se puedan extrapolar a otras poblaciones de similares características ${ }^{(30,31)}$.

Según datos recogidos de las historias clínicas las principales sustancias que motivaron la internación de los jóvenes fueron marihuana y pasta base de cocaína, seguida por clorhidrato de cocaína, alcohol, nicotina, alucinógenos, psicofármacos y bazuco.

Las entrevistas realizadas en forma individual fueron de duración menor que la prevista originalmente. Las respuestas fueron breves, con manifestaciones de aburrimiento y dificultad en elaborar las frases. En los grupos focales de 4 a 5 individuos cada uno, todos brindaron aportes respetando cada intervención. Las participaciones de algunos jóvenes resultaron más prolongadas y de otros más breves, dando lugar a respuestas y comentarios convergentes a los expresados en las entrevistas individuales.

Considerando la categoría "conocimiento" se encontraron los siguientes resultados sobre salud, enfermedad y funciones orales (Cuadro 2).

\begin{tabular}{|c|c|c|}
\hline Salud bucal & $\begin{array}{c}\text { Enfermedades } \\
\text { bucales }\end{array}$ & Funciones orales \\
\hline Dientes limpios & $\begin{array}{c}\text { Caries asociada a } \\
\text { dolor }\end{array}$ & Masticación \\
\hline Dientes blancos & Sangrado de encías & Estética \\
\hline Dientes alineados & $\begin{array}{c}\text { Enfermedades } \\
\text { transmisibles }\end{array}$ & Gusto \\
\hline
\end{tabular}

Cuadro 2. Categoría "conocimiento" y sus atributos o subcategorías

Considerando la categoría "higiene" se encontraron los siguientes resultados sobre realización, frecuencia y educación recibida (Cuadro 3).

\begin{tabular}{|l|c|c|c|}
\hline & Realización & Frecuencia & Aprendizaje \\
\hline Antes del consumo & De forma regular & Después de las comidas & $\begin{array}{c}\text { Instrucción recibida en la escuela, el hogar, en el } \\
\text { consultorio médico u odontológico. }\end{array}$ \\
\hline Después del consumo & De forma irregular & Cuando se acuerdan & No se reforzó la enseñanza \\
\hline
\end{tabular}

Cuadro 3. Categoría "higiene" y sus atributos o subcategorías

Considerando la categoría "relación con el entorno" se encontraron los siguientes resultados sobre influencia en trabajo y estudio, relación de pareja, relaciones familiares y con la sociedad antes y después de la situación de consumo (Cuadro 4). El conocimiento que los participantes de la investigación tenían sobre salud-enfermedad bucal se relacionó a la blancura, alineación correcta y limpieza de las piezas dentarias. Las enfermedades bucales más conocidas fueron caries dental, sangrado de las encías y enfermedades infecciosas transmisibles como HIV y sífilis. Las principales funciones orales alteradas según sus relatos fueron la masticación, la estética y el gusto a partir de la situación de consumo habitual de SPA.

Sobre higiene bucal se destacó que antes de la drogodependencia la realizaban en forma regular después de las principales comidas y que recibieron instrucciones de higiene buco-dental en sus casas, en la escuela o en consultorios médicos u odontológicos. A partir de la situación de dependencia la higiene pasó a ser irregular, a 
hacerla si se acordaban y que no se reforzaron sus conocimientos en ese tema.

Sobre la relación con el entorno antes del consumo problemático eran individuos en actividad escolar o laboral, con pareja o en procura de tenerla, que concurrían regularmente a centros de enseńanza y/o lugares de trabajo, a la consulta médica y odontológica. Después del consumo abusivo, manifestaron situaciones de automarginación por miedo al rechazo, baja autoestima, pérdida de pareja o contacto con los hijos, si los tuvieran, para evitar situaciones de violencia. También expresaron la opción de aislamiento de la familia para protegerla, situación de calle o vida en refugios, además de desistir de la asistencia a la consulta médica u odontológica al sentirse marginados o discriminados, con intentos de autoeliminación y conflictos legales que los pueden conducir a la privación de libertad.

En relación a la accesibilidad a la atención odontológica se reconocen diferencias antes y después de la adicción a SPA (Cuadro 5).
La accesibilidad a la consulta odontológica se realizaba en forma regular y criterio preventivo, eventualmente curativo, de preferencia en dependencias públicas o en la Facultad de Odontología, en épocas previas al consumo. Una vez generada la situación de dependencia a SPA, la atención pasó a ser esporádica en casos de urgencia, con terapias sedantes o quirúrgicas, muchas veces traumáticas para el paciente. Relatan deseos de mejorar su estado bucal aunque los costos y la escasez de recursos se lo impiden.

Los dibujos realizados bajo la supervisión de educadores, sin presencia de integrantes del equipo investigador, dieron éstos resultados. En el análisis de los dibujos se tomaron como referencia trabajos de Paul Ekman y Freitas- Magalhaes en cuanto a la interpretación de la sonrisa como lenguaje corporal que muestra "el alma interior" (32-33). Se distinguen tres grupos acerca de las imágenes: el primero con sonrisas de colores vivos en rojo, rosado o hasta con tintes de amarillo, mostrando bocas alegres, abiertas, con

\begin{tabular}{|c|c|c|c|c|}
\hline & $\begin{array}{c}\text { Influencia en trabajo y } \\
\text { estudio }\end{array}$ & Relación de pareja & $\begin{array}{l}\text { Relaciones fami- } \\
\text { liares }\end{array}$ & Relación con la sociedad \\
\hline $\begin{array}{l}\text { Antes del con- } \\
\text { sumo }\end{array}$ & $\begin{array}{c}\text { Integración al trabajo y/0 } \\
\text { estudio }\end{array}$ & $\begin{array}{c}\text { Solían tener o procurar } \\
\text { pareja }\end{array}$ & $\begin{array}{l}\text { Vida familiar } \\
\text { ordenada }\end{array}$ & $\begin{array}{l}\text { Concurren regularmente a centros } \\
\text { de enseñanza y/o lugares de } \\
\text { trabajo, a la consulta médica y } \\
\text { odontológica. }\end{array}$ \\
\hline $\begin{array}{l}\text { Después del } \\
\text { consumo }\end{array}$ & $\begin{array}{l}\text { Automarginación, } \\
\text { miedo al rechazo, baja } \\
\text { autoestima. }\end{array}$ & $\begin{array}{l}\text { Pérdida de pareja y de } \\
\text { contacto con los hijos. } \\
\text { Evitan situaciones de } \\
\text { violencia. }\end{array}$ & $\begin{array}{l}\text { Aislación para } \\
\text { proteger a la familia. } \\
\text { Situación de calle } 0 \\
\text { vida en refugios. }\end{array}$ & $\begin{array}{l}\text { Discriminación social, no estudian } \\
\text { ni trabajan, sólo consultas médicas } \\
\text { u odontológicas de urgencia, } \\
\text { antecedentes de conflictos legales, } \\
\text { intentos de autoeliminación. }\end{array}$ \\
\hline
\end{tabular}

Cuadro 4. Categoría “relación con el entorno” y sus atributos o subcategorías

\begin{tabular}{|c|c|}
\hline $\begin{array}{c}\text { Antes del con- } \\
\text { sumo }\end{array}$ & $\begin{array}{c}\text { Acceso a la consulta odontológica en } \\
\text { la niñez y adolescencia }\end{array}$ \\
\hline $\begin{array}{c}\text { Concurren al dentista por limpieza y } \\
\text { caries, recuerdan pocas experiencias } \\
\text { traumáticas o no las padecieron. }\end{array}$ \\
\hline $\begin{array}{c}\text { Después del } \\
\text { consumo }\end{array}$ & $\begin{array}{c}\text { No concurren excepto en situaciones } \\
\text { de urgencia. Recuerdo de extracciones } \\
\text { traumáticas. }\end{array}$ \\
\hline
\end{tabular}

Cuadro 5. Accesibilidad de la consulta odontológica

Limitaciones de la accesibilidad
No se asisten por no manifestar
dolor a sus padres 0 adultos
responsables.
Desconocen donde ir. Limitaciones
económicas para restauraciones
dentales o confección de prótesis.

Lugar de asistencia

Hospitales o policlínicas públicas de Montevideo o interior del país, Facultad de Odontología.

Pretenden concurrir a dependencias de ASSE, Facultad de Odontología o consulta privada 
presencia de dientes, incluso acompañada por flores como rosas.

Otro grupo mostró sonrisas más pequeñas, tristes, grises (en blanco y negro).

El tercer grupo también en blanco y negro con alguna tonalidad de rojo y verde. Se aprecian sonrisas con ojos, narices, corazones, elefantes, piernas, flores y lágrimas en el rostro cuando la boca está deteriorada.

Los dibujos de bocas con alteraciones expresan ausencias dentarias, caries representadas por manchas oscuras, pigmentaciones dentarias en color amarillo, labios agrietados.

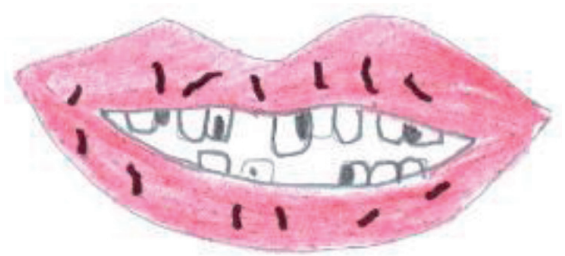

Fig. 1. Boca en salud y enfermedad según participante 1
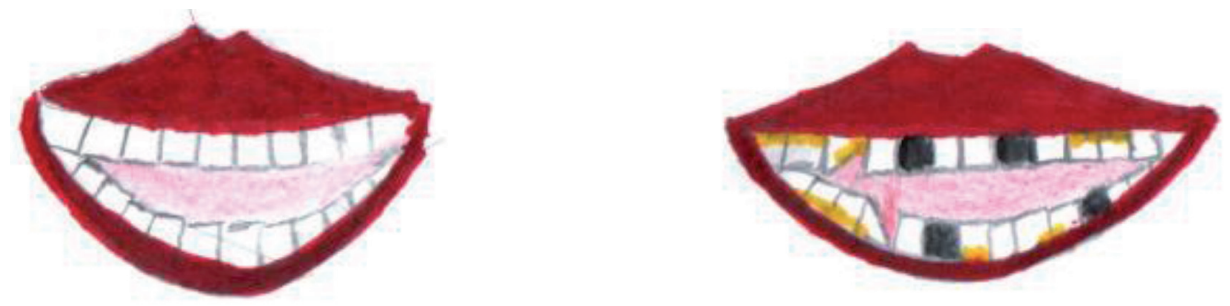

Fig. 2. Boca en salud y enfermedad según participante 2
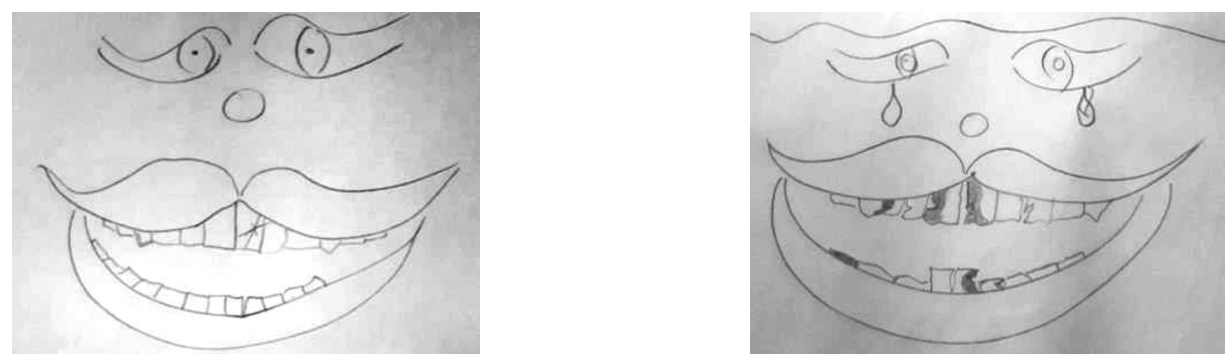

Fig. 3. Boca en salud y enfermedad en participante 3

\section{Discusión}

El estudio realizado cuyo análisis se basó en la teoría fundamentada, mediante la comparación constante, nos permitió llegar a resultados semejantes al de otros trabajos realizados en los cuales los individuos en tratamiento por drogodependencia perciben un deterioro de su salud bucal a partir del consumo de sustancias y por otro lado contrasta con estudios que analizan la percepción de jóvenes fuera de la drogodependencia.

Es así que los resultados de la población objeto a partir de la situación de dependencia difieren de investigaciones realizadas en Navarra, España en 2007 en niños y adolescentes escolarizados sin relato de consumo, quienes indicaron que la percepción subjetiva de salud bucodental 
era buena, la concurrencia a la consulta odontológica en el último año fue elevada, con más de un cepillado bucal diario y creencia de necesidad de algún tratamiento odontológico, como ortodoncia entre los adolescentes ${ }^{(34)}$.

Según otro estudio cualitativo realizado en Australia con pacientes en tratamiento por consumo, se coincide en que el conocimiento sobre acceso a servicios de salud bucal fue limitado y la búsqueda de asistencia ocurrió solo en situaciones de urgencia, sin la adecuada promoción de salud, al igual que en el presente trabajo ${ }^{(35)}$. Acorde un estudio multicéntrico realizado con población general mayor a 18 años no institucionalizada en las ciudades de Santiago, Tucumán y Porto Alegre, la frecuencia de cepillado, la higiene interdental y la consulta odontológica regular de carácter preventivo son importantes indicadores de salud gingival, mientras que el autoreporte de sangrado gingival es indicador de enfermedad, coincidiendo con declaraciones del presente estudio ${ }^{(36)}$.

Otro trabajo de investigación en el Estado de San Pablo, Brasil, coincide con el nuestro en el sentido que los adolescentes escolarizados entrevistados relacionaron su percepción de salud bucal con la higiene, estética o apariencia y visita regular al odontólogo ${ }^{(37)}$.

De acuerdo con estudios cuanti-cualitativos realizados en la ciudad de Tacuarembó del interior de nuestro país, también las consecuencias del uso de drogas fueron: problemas de salud general; conflictos familiares, vecinales y laborales; situaciones de inseguridad y violencia con el entorno social que podrían transgredir las normas legales ${ }^{(16)}$.

\section{Conclusiones}

Las personas jóvenes en tratamiento por dependencia a las drogas perciben el deterioro de su salud bucal a partir de la situación de consumo, alterando la estética, las funciones orales y la autoestima. El estado bucal afecta la calidad de vida de los jóvenes consumidores, quedando más vulnerables y discriminados en la sociedad. El conocimiento sobre la salud bucal que tienen estos jóvenes se limita a caries, gingivitis y enfermedades transmisibles vía oral, en particular infecciones de transmisión sexual.

En cuanto a las funciones orales afectadas las identifican principalmente con masticación, gustación y estética.

La asistencia odontológica se asoció a situaciones de urgencia en servicios públicos y en la Facultad de Odontología, sin cumplir controles periódicos. Sería conveniente proponer estrategias para que el profesional odontólogo y su equipo auxiliar les brinden una atención especial centrada en la prevención y promoción de salud a estas personas en conjunción y como parte del tratamiento por drogodependencia.

Agradecimientos: La investigación contó con la autorización de la Dirección del Portal Amarillo.

\section{Contribución de autoría}

1. Concepción y diseño del estudio

2. Adquisición de datos

3. Análisis de datos

4. Discusión de los resultados

5. Redacción del manuscrito

6. Aprobación de la versión final del manuscrito

ER ha contribuido en $1,2,3,4,5$ y 6 . SP ha contribuido en $1,2,3,4,5$ y 6 .

IS ha contribuido en 1,2 y 4 .

MTA ha contribuido en 1 y 2.

BF ha contribuido en 1 y 2.

KS ha contribuido en 1 y 2.

MCM ha contribuido en 1, 2 y 4 .

\section{Referencias}

1. WHO 2016. Organización Mundial de la Salud. Disponible en: http: //www.who.int/es/

2. Organización Panamericana de la Salud. Neurociencia del consumo y dependencia de sustan- 
cias psicoactivas [Internet]. Washington: Organización Mundial de la Salud; 2004. Disponible en: https: //www.who.int/substance_abuse/publications/neuroscience_spanish.pdf

3. Uruguay. Junta Nacional de Drogas. Drogas: más información menos riesgos. $11^{\mathrm{a}} \mathrm{ed}$. Montevideo: IMPO; 2016.

4. CEF. Comité de Expertos de la OMS en Farmacodependencia: Serie de informes técnicos, No 915-33-Informe-OMS (2003). Disponible en: apps.who.int/medicinedocs/es/d/Js4898s/

5. Pereira T. Neurobiología de la adicción. Rev Psiquiatr Urug. 2008; 73 (1): 9-24.

6. OEA. Organización de los Estados Americanos. El problema de las drogas en las Américas: Estudios drogas y salud pública. Washington: Organización de los Estados Americanos; 2013. Disponible en: http: //www.cicad.oas.org/drogas/elinforme/informeDrogas2013/drugsPublicHealth_ESP.pdf

7. UNODC. Oficina de las Naciones Unidas contra la Droga y el Delito. Resumen, conclusiones y consecuencias en materia de políticas: Informe Mundial sobre las Drogas 2017. Viena: UNDOC; 2017. Disponible en: https: //www. unodc.org/wdr2017/field/WDR_Booklet1_ Exsum_Spanish.pdf

8. Sánchez Antelo V. La dimensión temporal del consumo de drogas: análisis sociológico desde una categoría clave para el estudio de los procesos de salud-enfermedad-atención-cuidado. Salud Colec. 2016; 12 (1): 41-54.

9. Berruecos Villalobos L. Drogadicción, farmacodependencia y drogodependencia: definiciones, confusiones y aclaraciones. Cuicuilco. 2010; 17 (49): 61-81.

10. UNODC. Oficina de las Naciones Unidas contra la Droga y el Delito. Informe Mundial sobre las Drogas 2016. New York: UNDOC; 2016. Disponible en: https: //www.unodc.org/doc/ wdr2016/V1604260_Spanish.pdf

11. Toledo M. Perfil clínico epidemiológico de adolescentes consumidores de drogas en el Hospital Hermilio Valdizan. Rev. de Psiqu. Salud Mental Hermilio Valdizan. 2003; 4 (1): 19-28.

12. Ortega-Pérez CA, da Costa-Júnior ML, Pereira Vasters G. Perfil epidemiológico de la drogadic- ción en estudiantes universitarios. Rev. LatinoAm. Enfermagem. 2011; 19: 665-72.

13. Degenhardt L, Peacock A, Colledge S, Leung J, Vickerman P. Global prevalence of injecting drug use and sociodemographic characteristics and prevalence of HIV, HBV and HCV in people who inject drugs: a multistage systematic review. The Lancet Global Healt. 2017; 5 (12): e 1192- e1207. Disponible en: www.thelancet. com/lancetgh vol5 december 2017

14. Uruguay. JND. Junta Nacional de Drogas 2016. VII Encuesta nacional sobre consumo de drogas en estudiantes de Enseñanza Media -2016 https: //www.gub.uy/jnd/comunicacion/ publicaciones/vii-encuesta-nacional-sobre-consumo-de-drogas-en-estudiantes-de

15. Uruguay. JND. Junta Nacional de Drogas. (2016) VI Encuesta Nacional en Hogares sobre Consumo de Drogas. Disponible en: https: // www.gub.uy/junta-nacional-drogas/sites/juntanacional-drogas/files/documentos/publicaciones/201609_VI_encuesta_hogares_OUD_ultima_rev.pdf

16. Steffano D. Diagnóstico sobre el consumo de drogas en la zona del centro de la ciudad de Tacuarembó-Departamento de Tacuarembó. CICAD- OEA. JND. Junta Departamental de Drogas de Tacuarembó.

17. UNODC. Oficina de las Naciones Unidas contra la Droga y el Delito. Informe Mundial sobre las Drogas 2009. Nueva York: UNDOC; 2009. Disponible en: https: //www.unodc.org/documents/wdr/WDR_2009/World_Drug_Report_2009_spanish.pdf

18. Muñoz Astudillo MN, Gallego Cortes C, Wartski Patino CI, Álvarez Sierra LE. Familia y consumo de sustancias psicoactivas: una búsqueda de lo ausente. Index Enferm [online]. 2012; 2 (3): 136-140. Disponible en: <http: //scielo.isciii. es/scielo.php?script=sci_arttext\&pid=S1132$12962012000200006 \& \operatorname{lng}=e s \& n r m=i s o>$.

19. AAPD. American Academy of Pediatric Dentistry. Policy on substance abuse in adolescent patients. Reference manual. 2016; 39 (6): 17-18.

20. Barrios CE, Vila VG. Estado de salud bucodental en consumidores de marihuana. Rev. Fac. Odon. 2009; 2 (1): 30-35. 
21. Brown C, Krishnan S, Hursh K, Yu M, Johnson P, Page K, Shiboski CH. Dental disease prevalence among methamphetamine and heroin users in an urban setting: a pilot study. J Am Dent Assoc. 2012; 143: 992-1001.

22. Gigena PC, Bella MI, Cornejo LS. Salud bucal y hábitos de consumo de sustancias psicoactivas en adolescentes y jóvenes drogodependientes en recuperación. Odontoestomatología. 2012; 14 (20): 49-59.

23. Gupta T, Shah N, Mathur VP, Dhawan A. Oral health status of a group of illicit drug users in Delhi, India. Community Dental Health. 2012; 29(1): 49-54.

24. Rotemberg E, Salveraglio I, Piovesán S, Kreiner M, Smaisik K, Ormaechea R, Varela A. Estado dental y periodontal de población en tratamiento por consumo de drogas. Estudio piloto. Odontoestomatología. 2015; 17 (25): 34-39.

25. Chaparro-González NT, Fox-Delgado MA, Pineda-Chaparro RT, Perozo-Ferrer BI, DíazAmell AR, Torres-Quintero VC. Manifestaciones bucales y maxilofaciales en pacientes con adicción a las drogas. Odontoestomatología. 2018; 20 (32): 24-31.

26. Barnetche MM, Cornejo LS. Calidad de vida y proceso salud enfermedad atención a nivel del componente bucal de jóvenes alojados en centros socioeducativos del Área Penal Juvenil de la Provincia de Córdoba. Tesis de Doctorado. Facultad de Odontología. Universidad Nacional de Córdoba, 2017.

27. FDI. Federación Dental Internacional. Salud buco-dental y calidad de vida. Bankok: FDI; 2015. Disponible en: https: //www. fdiworlddental.org/es/resources/policy-statements-and-resolutions/salud-bucodental-y-calidad-de-vida

28. FDI. Federación Dental Internacional. FDI's definition of oral health. Enero, 2019. Disponible en: https: //www.fdiworlddental.org/oralhealth/fdi-definition-of-oral-health

29. Díaz- Cárdenas S, Meisser-Vidal MA, TiradoAmador LR, Fortich-Mesa N, Tapias-Torrado
L, González-Martínez FD. Impacto de salud oral sobre calidad de vida en adultos jóvenes de clínicas odontológicas universitarias. Int. J. Odontostomat. 2017; 11 (1): 5-11.

30. Triaca J, Cardeillac V, Idiarte Borda C. Características de los primeros usuarios que consultaron en el Centro de Referencia Nacional de la Res Drogas Portal Amarillo. Rev Psiquiatr Urug. 2009; 73 (1): 37-48.

31. Riva R, Rotemberg E, Sanguinetti M, Rodríguez A, Massa F. Drogodependencia, Bruxismo y Trastornos Témporo-Mandibulares. Análisis comparativo en dos poblaciones: muestra nacional y población en tratamiento por consumo problemático de drogas en Portal Amarillo Odontoestomat. 2014; 16 (24): 26-33.

32. Ekman P \& Friesen WV. Manual for the Facial Action Code. Palo Alto, CA: Consulting Psychologist Press; 1978.

33. Freitas-Magalhães A (2012). Facial expression of emotion. In V. S. Ramachandran (Ed.), Encyclopedia of Human Behavior Vol. 2: 173-183.

34. Artazcóz J, Cortés FJ, Rosel E, González Rodríguez P, Bravo M. Percepción y hábitos de salud bucodental en niños y adolescentes de Navarra, 2007. An Sist Sanit Navar. 2010; 33 (1): 51-64.

35. Cheah, Alison Li Sun; Pandey, Ram; Daglish, Mark; Ford, Pauline J; Patterson, Sue. A qualitative study of patients' knowledge and views of about oral health and acceptability of related intervention in an Australian inpatient alcohol and drug treatment facility. Health Soc Care Community. 2017; 25(3): 1209-1217.

36. Gómez MV, Toledo A, Carvajal P, Gomes SC, Costa RSA, Solanes F, Oppermann SV, Rosing CK, Gamonal J, Romanelli H. A multicenter study of oral health behavior among adult subject from three South American cities. Braz. Oral Res. 2018; 32: e22.

37. Saliba-Garbin CA, Isper-Garbin AJ, MoreiraArcieri R, Saliba NA, Goncalves PE. La salud bucal en la percepción del adolescente. Rev Salud Pública. 2009. 11 (2): 268-277.

\section{Enrique Rotemberg: erotemb@yahoo.com.ar}

